\title{
Entanglement entropy in quasi-symmetric multi-qubit states
}

\author{
Zhi-Hua Li and An-Min Wang \\ Department of Modern Physics, University of Science \\ and Technology of China, Hefei 230026, China
}

\begin{abstract}
We generalize the symmetric multi-qubit states to their $q$-analogs, whose basis vectors are identified with the $q$-Dicke states. We study the entanglement entropy in these states and find that entanglement is extruded towards certain regions of the system due to the inhomogeneity aroused by $q$-deformation. We also calculate entanglement entropy in ground states of a related $q$-deformed Lipkin-Meshkov-Glick model and show that the singularities of entanglement can correctly signify the quantum phase transition points for different strengths of $q$-deformation.
\end{abstract}

\section{INTRODUCTION}

Quantum entanglement as the resource of quantum information process is the genuine characteristic of quantum systems [1]. In the last decade, there have been enormous interests in studying entanglement of quantum many body systems. The Hilbert space $\left(\mathbb{C}^{2}\right)^{\otimes N}$, taking $N$-qubit systems for example, grows exponentially large with $N$, so measuring of entanglement of arbitrary states in $\left(\mathbb{C}^{2}\right)^{\otimes N}$ is generally hard [2]. One simpler task is to restrict to some particular subsets of $\left(\mathbb{C}^{2}\right)^{\otimes N}$, in which the computation of entanglement should be feasible. An example is the symmetric subspace, which is spanned by the states obtained by superposition of distinct permutations of state like $|11 \cdots 10 \cdots 0\rangle$ with $k$ spin-up's and $(N-k)$ spin-down's. Pairwise entanglement and entanglement entropy have been obtained in these states [3, 4]. Since the early works, the symmetric states have become a testing ground for new entanglement measures [5-7] and for elucidating some features of entanglement structure in many-particle systems [2, 8, 9]. The computation of entanglement in these states can also be used in the studies of quantum spin chain models whose Hamiltonian has the permutation invariance, an example being the Lipkin-Meshkov-Glick (LMG) model [10]. In regard to the seminal works on the intriguing relations between entanglement and quantum phase transition [11 15] (see [16] for a review), the relation of anomaly of ground state quantum entanglement with quantum phase transition in the LMG model have also been 
established [17]22].

Given that the symmetric states are amenable to the characterization of entanglement properties of quantum many-body systems and that they can be realized in experiments [23, 24], it is theoretically desirable to extend these entanglement-computable states to a wider range for further analysis. In physics, one particular way to extend certain physical models or states is the quantum deformation through quantum groups [25 27]. Recently there have been some interests in studying entanglement in $q$-deformed many body systems, such as the $q$-deformed coherent states[28, 29], $q$-VBS states [30, 31], $q$-Werner states[32] and some related models. The $q$-deformed states usually exhibits some non-classical properties which provide new perspectives on the understanding of entanglement in many body systems and may have potential applications in certain quantum information tasks.

In this work we extend the symmetric states to a wider class of states depending on a deformation parameter $q$, whose basis is later identified with the $q$-Dicke states through the $\mathrm{SU}_{q}(2)$ quantum group. The deformation breaks the permutation invariance in the original symmetric states, but we show that the requirement of permutation invariance can be actually to some extent relaxed in the calculation of entanglement entropy. Besides, these states don't even preserve the cyclic or reflection invariance. As a result, we find that entanglement is extruded to certain regions of the system. This behaviour differs from the usual many body states studied in the literature where the entropy are mostly found to be symmetric about the middle bipartition. In addition we also study a corresponding $q$-deformed LMG model. Surprisingly, the singularity in the entanglement entropy can still correctly signify quantum phase transition even in the presence of $q$-deformation.

\section{II. q-ANALOG OF SYMMETRIC STATES}

In order to define the $q$-analog of symmetric states, let us recall the notations of $q$-analogs of some usual mathematical objects: The $q$-factorial is

$$
[n] !=[n][n-1] \cdots[1]
$$

where

$$
[x] \equiv \frac{q^{x}-q^{-x}}{q-q^{-1}}
$$


denotes the $q$-numbers and $q>0$. $[x]$ is invariant when change $q \leftrightarrow q^{-1}$. Then the $q$-binomial coefficients are

$$
\left[\begin{array}{l}
n \\
m
\end{array}\right]=\frac{[n] !}{[m] ![n-m] !}
$$

In the limit of $q \rightarrow 1$, the above definitions all return to the normal ones: $[x] \rightarrow x,[n] ! \rightarrow n !$ and $\left[\begin{array}{c}n \\ m\end{array}\right] \rightarrow\left(\begin{array}{c}n \\ m\end{array}\right)$.

Now we consider the following classes of states $|N, k\rangle_{q} \in\left(\mathbb{C}^{2}\right)^{\otimes N}, k=0,1, \cdots N$, which are extensions of the basis of symmetric states for each $q>0$

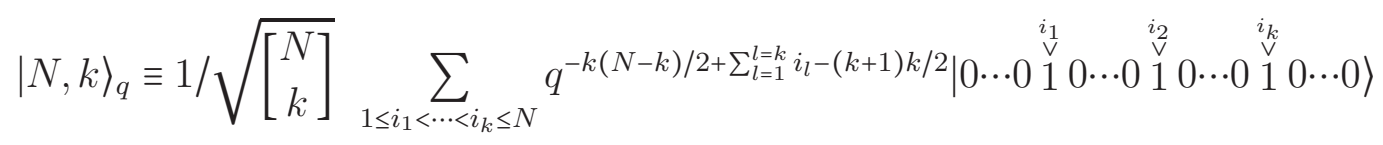

They include basis of the symmetric states for $q=1$. Largely speaking each state $|N, k\rangle_{q}$ is a superposition of all distinct permutations of the state $\left|1 \cdots 1_{k} 0 \cdots 0_{N}\right\rangle$ with nontrivial coefficients which are $q$ 's powers (Let alone the overall normalization factor $1 / \sqrt{\left[\begin{array}{c}N \\ k\end{array}\right]}$ ). The powers of the $q$-factors for each permutations are seen being defined in the following rules: 1) Starting from the "word" $11 \cdots 100 \cdots 0$ with $\mathrm{k} 1$ 's followed by $(N-k) 0$ 's, its $q$-factor is set $\left.q^{-k(N-k) / 2} ; 2\right)$ Each time a 1 crosses over a 0 from left to right will produce a factor $q$. From these, as for a generic word having 1's at positions $i_{1}, i_{2}, \cdots, i_{k}$, the power on $q$ is $-k(N-k) / 2+\sum_{l=1}^{l=k}\left(i_{l}-l\right)$, besides, the squares of these $q$-factors sum up to $1 /\left[\begin{array}{l}N \\ k\end{array}\right]$, thus finally yielding the properly normalized form of eq.(44). One may also put the rules simply: The more 1's distributed on the rightmost positions in the word, the larger the power on $q$ [33]. These rules are most easily anticipated by looking at a simple example, e.g.,

$$
|4,1\rangle_{q}=1 / \sqrt{[4]}\left(q^{-3 / 2}|1000\rangle+q^{-1 / 2}|0100\rangle+q^{1 / 2}|0010\rangle+q^{3 / 2}|0001\rangle\right),
$$

with [4] $=q^{-3}+q^{-1}+q^{1}+q^{3}$. The $q$-factors in essence keep track of how the 1's and 0's are permuted and can be regarded as inhomogeneous weights for each permuted words. As a result, each states $|N, k\rangle_{q}$ are no longer permutation invariant, thus we may also call them and all of their superpositions the quasi-symmetric states. Additionally, we stress that the $q$-factors even break in special the cyclic subgroup and reflection subgroup of the symmetric group. This inhomogeneity will cause unusual behaviours in bipartite entanglement.

The above definition of quasi-symmetric states is combinatorics flavored. Actually they are identified with the $q$-Dicke states within the $\mathrm{SU}_{q}(2)$ quantum group formalisms. The algebraic approach will facilitate later the calculation of the entanglement property of the states and the definition of related physical models. 
Recall the definition of the $\mathrm{SU}_{q}(2)$ algebra, which is generated by the operatoers $S^{+}, S^{-}$ and $S^{z}$ subject to relations:

$$
\begin{aligned}
& {\left[S^{z}, S^{ \pm}\right]=S^{ \pm}} \\
& {\left[S^{+}, S^{-}\right]=\left[2 S_{z}\right] \equiv \frac{q^{2 S^{z}}-q^{-2 S^{z}}}{q-q^{-1}},}
\end{aligned}
$$

Its representations are in parallel with $\mathrm{SU}(2)$ algebra[26] : Each irreducible representation is labeled by the total $q$-spin $S \in\left\{0, \frac{1}{2}, 1, \frac{3}{2}, \cdots\right\}$. For a fixed $S$, the representation space $V^{S}$ is of dimension $2 S+1$ and spanned by the so called $q$-Dicke states $\left\{|S, M\rangle_{q}, M=-S,-S+1, \cdots, S\right\}$ (Here using $|\cdot\rangle\rangle$ to avoid confusion with the notation in eq.(44)). The representation matrices of the generators are given through:

$$
\begin{aligned}
& \left.\left.S^{z}|S, M\rangle\right\rangle_{q}=M|S, M\rangle\right\rangle_{q} \\
& \left.\left.S^{ \pm}|S, M\rangle\right\rangle_{q}=\sqrt{[S \mp M][S \pm M+1]}|S, M \pm 1\rangle\right\rangle_{q}
\end{aligned}
$$

In particular, the fundamental representation for the $\mathrm{SU}_{q}(2)$ algebra is trivially identical to that of the $\mathrm{SU}(2)$ algebra: The representation space is $V^{\frac{1}{2}} \equiv\{|1\rangle,|0\rangle\}(|1\rangle$ and $|0\rangle$ are spin up and down respectively), and the representation matrices are $s^{z} \equiv \frac{1}{2} \sigma^{z}, s^{ \pm} \equiv \sigma^{x} \pm i \sigma^{y}$, with $\sigma^{\alpha}, \alpha=x, y, z$ being Pauli matrices.

A well known fact about $\mathrm{SU}(2)$ Lie algebra is that, all its irreducible representations can be constructed by coupling of several spin-1/2 representation matrices. Similar situation also holds for the $\mathrm{SU}_{q}(2)$ algebra. This fact entails connection between formalisms of Lie algebras or quantum algebras with the multi-qubit systems, so we restate this fact in detail. The only subtlety in the $\mathrm{SU}_{q}(2)$ case is that in order that the coupled $q$-spins to respect the $\mathrm{SU}_{q}(2)$ relations ([6), one must utilize the comultiplication $\Delta: S U_{q}(2) \rightarrow S U_{q}(2)^{\otimes 2}$,

$$
\begin{aligned}
& \Delta\left(S^{z}\right)=S^{z} \otimes 1+1 \otimes S^{z} \\
& \Delta\left(S^{ \pm}\right)=q^{S^{z}} \otimes S^{ \pm}+S^{ \pm} \otimes q^{-S^{z}}
\end{aligned}
$$

Then the coupling of $N q$-spin-1/2 operators are obtained by acting $\Delta$ on $s^{ \pm, z}$ for $N$ times:

$$
\begin{aligned}
& \tilde{S}^{z}=\Delta^{N}\left(s^{z}\right)=\sum_{i=1}^{N} 1 \otimes \cdots \otimes 1 \otimes \stackrel{\stackrel{i}{\vee}}{z} \otimes 1 \otimes \cdots \otimes 1 \\
& \tilde{S}^{ \pm}=\Delta^{N}\left(s^{ \pm}\right)=\sum_{i=1}^{N} q^{s_{z}} \otimes \cdots \otimes q^{s_{z}} \otimes \stackrel{\stackrel{i}{\vee}}{\sigma^{ \pm}} \otimes q^{-s_{z}} \otimes \cdots \otimes q^{-s_{z}}
\end{aligned}
$$

so that $\tilde{S}^{ \pm, z}$ are some particular representation matrices for $S^{ \pm, z}$. Next is to determine which representation the operators $\tilde{S}^{ \pm, z}$ belong to: First note the state $|N, 0\rangle_{q}=\left|0_{1} 0_{2} \cdots 0_{N}\right\rangle$ is the 
lowest weight vector for this representation as $\tilde{S}^{-}|N, 0\rangle_{q}=0$. Successive action on $|N, 0\rangle_{q}$ by $\tilde{S}^{+}$for $k$ times $k=0,1,2 \cdots$ will generate a whole basis for this representation, which can be calculated directly by using

$$
\begin{aligned}
& \tilde{S}^{+k}=[k] ! \sum_{1 \leq i_{1}<i_{2}<\cdots<i_{k} \leq N} q^{k s_{z}} \otimes \cdots \otimes q^{k s_{z}} \otimes \sigma^{\stackrel{i_{1}}{i^{\prime}}} \otimes q^{(k-2) s_{z}} \otimes \cdots \otimes q^{(k-2) s_{z}} \\
& \otimes \stackrel{i_{2}}{\stackrel{i_{2}}{\sigma^{+}}} \otimes q^{(k-4) s_{z}} \otimes \cdots \otimes \sigma^{+} \stackrel{\substack{i_{k} \\
\sigma^{+}}}{-k s_{z}} \otimes \cdots \otimes q^{-k s_{z}}
\end{aligned}
$$

The result of the calculation turns out to be that $\tilde{S}^{+k}|N, 0\rangle_{q}$ is nothing but expressions of the quasi-symmetric states $|N, k\rangle_{q}$ (up to a normalization factor) for $k \leq N$ and equals 0 when $k>N$. So the dimension of this representation is $N+1$, and considering the uniqueness of the irreducible representation of $\mathrm{SU}_{q}(2)$ [26], this determines the representation to be $S=N / 2$. Finally, according to (7) we conclude the identification of the quasi-symmetric states with the $q$-Dicke states $\left.|N, k\rangle_{q} \equiv|S=N / 2, M=k+N / 2\rangle\right\rangle_{q}$. With this identification, we can utilize the $\mathrm{SU}_{q}(2)$ algebra to calculate entanglement properties of the quasi-symmetric states and construct models that generate these states, as shown in following sections.

\section{BIPARTITE ENTANGLEMENT OF QUASI-SYMMETRIC STATES}

A generic $N$ qubit system can be split in a variety of ways. In this paper we only consider bipartition of the system to $A$ and $B$ subsystems: $A=\{1,2, \cdots, L\}$ and $B=\{L+1, \cdots, N\}$ for $L=0,1, \cdots N$. Then to compute the entanglement entropy $(E E)$ of the states $|\psi\rangle \in\left(\mathbb{C}^{2}\right)^{\otimes N}$, one way is to get the Schmidt decomposition:

$$
|\psi\rangle=\sum_{i} \lambda_{i}\left|\psi_{A, i}\right\rangle \otimes\left|\psi_{B, i}\right\rangle
$$

where $\psi_{A, i} \in\left(\mathbb{C}^{2}\right)^{\otimes L}$ and $\psi_{B, i} \in\left(\mathbb{C}^{2}\right)^{\otimes(N-L)}$. These vectors coincide with the eigenvectors of the corresponding reduced density matrix $\rho_{A / B}=\operatorname{tr}_{B / A}(|\psi\rangle)=\sum_{i} \lambda_{i}^{2}\left|\psi_{A / B, i}\right\rangle\left\langle\psi_{A / B, i}\right|$. So the entanglement entropy is

$$
S_{N, L}=\sum_{i} \lambda_{i}^{2} \log _{2}\left(\lambda_{i}^{2}\right)
$$

In the computation of the bipartite entanglement of the symmetric states, their Schmidt decomposition is mapped from the decomposition of the corresponding Dicke states [17, 34]. The quasi-symmetric states can be computed in the same approach, except for replacing 

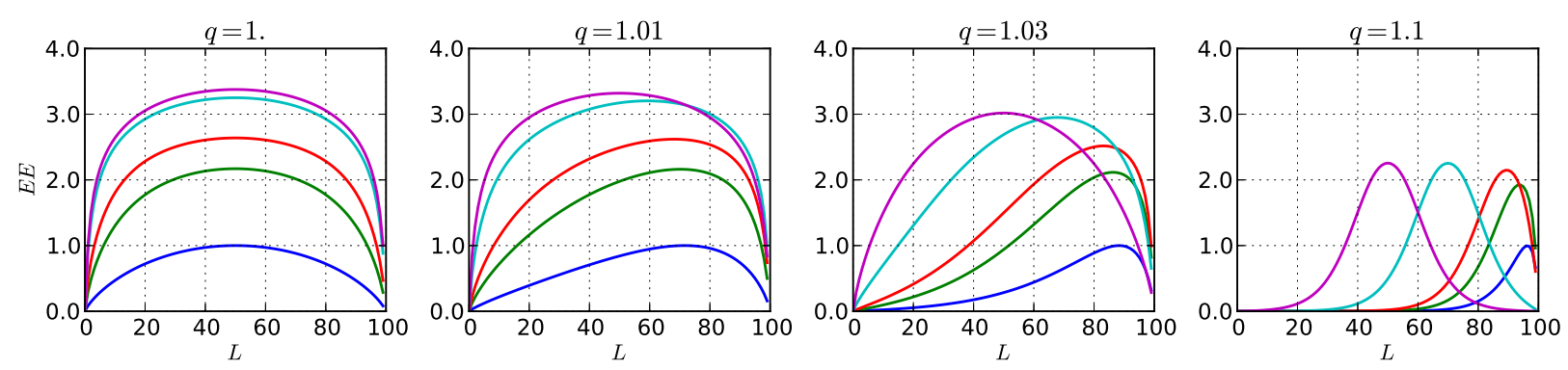

FIG. 1: Dependence of entanglement in $|N, k\rangle_{q}$ on the position of bipartition $L$ for several values of $q$ and $k$ : In each panel $N=100$ and (the left side of) the curves from bottom to top are $k=1,5,10,30,50$.

everything with their $q$-analogs: The decomposition of the $q$-Dicke states $F: V^{S} \rightarrow V^{\mu} \otimes V^{S-\mu}$ is just the decomposition of $q$-spin angular momentum, given by

$$
\left.\left.|S, M\rangle\rangle_{q}=\sum_{\nu=-\mu}^{\mu} C_{\nu, M-\nu, M}^{\mu, S-\mu, S}|\mu, \nu\rangle\right\rangle_{q} \otimes|S-\mu, M-\nu\rangle\right\rangle_{q},
$$

where $C$ is the $q$-Clebsh-Golden coefficients and its explicit expression (written in the standard notation) is [35]

$$
\begin{aligned}
C_{m_{1}, m_{2}, m}^{j_{1}, j_{2}, j_{1}+j_{2}}=\delta_{m_{1}+m_{2}, m} & q^{j_{1} m_{2}-j_{2} m_{1}} \\
\times & \left.\times \frac{\left[2 j_{1}\right] !\left[2 j_{2}\right] !\left[j_{1}+j_{2}+m\right] !\left[j_{1}+j_{2}-m\right] !}{\left[2 j_{1}+2 j_{2}\right] !\left[j_{1}-m_{1}\right] !\left[j_{1}+m_{1}\right] !\left[j_{2}-m_{2}\right] !\left[j_{2}+m_{2}\right] !}\right\}^{1 / 2}
\end{aligned}
$$

With the identification of $q$-Dicke states for $S \rightarrow N / 2, M \rightarrow k-N / 2, \mu \rightarrow L / 2$ and $\nu \rightarrow l-L / 2$, eq.(13) is mapped to the corresponding decomposition in the $N$ qubit system $\tilde{F}:\left(\mathbb{C}^{2}\right)^{\otimes N} \rightarrow$ $\left(\mathbb{C}^{2}\right)^{\otimes L} \otimes\left(\mathbb{C}^{2}\right)^{\otimes(N-L)}$, given by

$$
|N, k\rangle_{q}=\sum_{l=0}^{L} p_{N, k, q, L, l}^{1 / 2}|L, l\rangle_{q} \otimes|N-L, k-l\rangle_{q},
$$

where the Schmidt coefficients $p_{N, k, q, L, l}^{1 / 2}$ are translated from the $q$-CG coefficients in eq.(13):

$$
p_{N, k, q, L, l} \equiv C_{l-L / 2, k-l-(N-L) / 2, k-N / 2}^{L / 2,(N-L) / 2, N / 2}=q^{k L-N l}\left[\begin{array}{c}
N \\
l
\end{array}\right]\left[\begin{array}{c}
N-L \\
k-l
\end{array}\right] /\left[\begin{array}{c}
N \\
k
\end{array}\right]
$$

Note that $p$ is also the $q$-hypergeometric distribution and it has the symmetries $p_{N, k, q, L, l}=$ $p_{N, N-k, 1 / q, L, L-l}=p_{N, k, 1 / q, N-L, k-l}$. In the following we shall write $p_{q, l}$ as a shorthand for $p_{N, k, q, L, l}$ when its meaning is clear from the context. 
Once the Schmidt coefficients are obtained, the entropy is directly calculated through eq.(12). We numerically evaluate the summation in the entropy $S_{N, k, q}(L)=-\sum_{l=0}^{L} p_{q, l} \log _{2} p_{q, l}$ for a system with 100 spins as shown in Fig,1. Note the symmetries in $p_{q, l}$ in turn leads to symmetries in $E E: S_{N, k, 1 / q}(L)=S_{N, k, q}(N-L)$ and $S_{N, N-k, q}(L)=S_{N, k, q}(N-L)$, so that, in Fig, we have let $L$ vary fully from 0 to $N$ but select several values for $k$ and $q$ only in the ranges $k \leq N / 2$ and $q \geq 1$, respectively. Several properties can be observed: 1) For a fixed $N$ and $k$-deformation won't increase the maximal values of entanglement that can be reached. And, as $q$ increase, the amount of entanglement is depressed in the whole system. (Note in the limit $q \rightarrow \infty$, eq. (4) approaches a product state and entanglement vanishes); 2) The main effect of $q$-deformation on entanglement is that, as $q$ increases from 1 , entanglement is extruded towards one side of the system. And this effect is more obvious when $k$ deviates more from $N / 2$. The asymmmetry of the bipartite entanglement is mainly due to the inhomogeneity in $|N, k\rangle_{q}$. Nevertheless, note that changing $q \rightarrow q^{-1}$ amounts to the reflection operation on $|N, k\rangle_{q}$. So, in the figure the curves are extruded to the right, but if we change $q \rightarrow q^{-1}$ the curves would be just reflected about the line $L=50$ and thus towards the left side (This is also true if we change $k \rightarrow N-k$ ). We have explored much larger values of $N$ and other combinations with $k$ and $q$ and find that these a few examples have already captured the essential entanglement properties in these classes of states.

Having obtained the bipartite entanglement of each $|N, k\rangle_{q}$, one may consider any of their superposition states:

$$
|\psi\rangle_{q}=\sum_{k=0}^{N} \alpha_{k}|N, k\rangle_{q}
$$

For the bipartition of each $L$ as discussed above, the reduced density operator for $|\psi\rangle_{q}$ is readily written as

$$
\rho_{L}=\sum_{l, l^{\prime}=0}^{L} \sum_{k=0}^{N} \alpha_{k} \bar{\alpha}_{k-l+l^{\prime}} p_{q, l}^{1 / 2} p_{q, l^{\prime}}^{1 / 2}|L, l\rangle\left\langle l^{\prime}, L\right|,
$$

which corresponds to a $(L+1) \times(L+1)$ matrix. So for $L \sim O\left(10^{3}\right)$, one may numerically diagonalize it to obtain its eigenvalues and then the entanglement entropy. Instead of studying entanglement entropy of some generic quasi-symmetric quantum states, we calculate entanglement of the ground states in a $q$-deformed LMG model and emphasize relation between entanglement and quantum phase transition even in presence of $q$-deformation. 


\section{GROUND STATE ENTANGLEMENT IN $q$-DEFORMED LMG MODEL}

Avancini et. al. [36] have proposed a $q$-deformed LMG model:

$$
H_{q}=\frac{h}{4 \sinh (\gamma / 2)} \sinh \left(2 \gamma \tilde{S}_{0}\right)+\frac{\lambda}{2[N]}\left(\tilde{S}^{+2}+\tilde{S}^{-2}\right)
$$

where $\tilde{S}^{ \pm, z}$ are given by eq.(9) and $\gamma=\ln q$. When $q=1$ it is the isotropic LMG model

$$
H_{1}=h \sum_{i} \sigma_{i}^{z}+\frac{\lambda}{N} \sum_{i<j} \sigma_{i}^{+} \sigma_{j}^{+}+\sigma_{i}^{-} \sigma_{j}^{-}
$$

When $q \neq 1, H_{q}$ describe highly inhomogeneous systems, which are no longer permutation

invariant nor even translation invariant. Nevertheless, since $H_{q}$ can be written in terms of the total $q$-spin operators, it obviously has the $\mathrm{SU}_{q}(2)$ symmetry, i.e. the Casimir element of $\mathrm{SU}_{q}(2)$ algebra $C \equiv S^{-} S^{+}+\left[S^{z}+\frac{1}{2}\right]^{2}-\left[\frac{1}{2}\right]^{2}$ commutes with $H_{q}$ :

$$
\left[H_{q}, C\right]=0
$$

So the total $q$-spin is conserved and $H_{q}$ is block diagonal in each total $q$-spin sector. It is known that the ground state belongs to the $S=N / 2$ sector spanned by $\left.\left.\left.\{|N / 2,-N / 2\rangle\rangle_{q},|N / 2,-N / 2+1\rangle\right\rangle_{q}, \cdots,|N / 2, N / 2\rangle\right\rangle_{q}\right\}$. One may constraint $H_{q}$ in this sector and represent it by a $(N+1) \times(N+1)$ matrix under this basis. Then, for systems with number of spins $N \sim O\left(10^{3}\right)$, which is large enough to extract the thermodynamic limit properties, one can directly diagonalize the Hamiltonian to get the exact ground state $|\psi\rangle_{g s}$.

Since the ground state is a generic quasi-symmetric state, the bipartite entanglement can be calculated through eq.(17), (18) and (12). We numerically calculate the ground state and its entanglement in this approach. Without loss of generality, we fix $\lambda=1$ and let $H_{q}$ varies with $h$ and $q$. Bipartite entanglement with $N=1000, L=500$ for $|\psi\rangle_{g s}$ as a function of $h$ for different $q$ is shown in Fig. 2(a). One can see that, for each $q$, the entanglement has a cusp at some value of $h$, which we denote by $h_{c}$. We have tested other bipartition by adjusting $L$ and find the results are qualitatively similar. With respect to previous studies of entanglement in LMG model [17], one may expect that each cusp at $h_{c}$ indicates a quantum phase transition point. Note that $h_{c}$ decreases as $q$ increases. This behaviour is consistent with the result of [36] that increase $q$ will depress the phase transition. Furthermore in [36] 

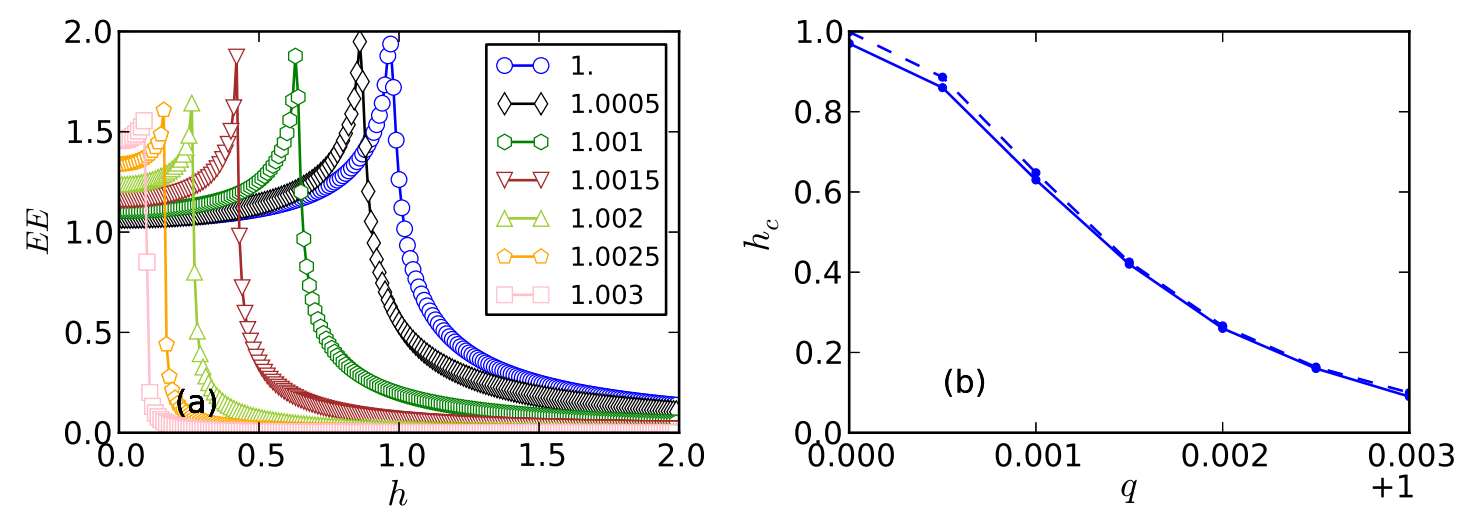

FIG. 2: (a) Dependence of bipartite entanglement $S_{N, L}\left(\left|\psi_{g s}\right\rangle\right)$ on magnetic field $h$ for different values of $q$, with $N=1000, L=500$. (b) Critical magnetic field $h_{c}$ as a function of $q$ : Solid line is determined through entanglement calculation; dashed through variation method.

the dependence of $h_{c}$ on $q$ has been obtained analytically through mean field variational studies, which can be written as

$$
h_{c}(q)=\frac{[N-1]}{[N]} \frac{2\left[\frac{1}{2}\right]}{1+2 \sinh \left[\frac{\gamma}{2}(N-1)\right]^{2}}
$$

We extract the dependence of $h_{c}$ on $q$ for several $q$ values from the above entanglement calculations and compare it with eq.(22), as shown in Fig.2(b). It is shown that these two rather different approaches match well. So these two results confirm each other and this is a strong evidence that $h_{c}$ are indeed quantum phase transition points. So we conclude that the cusp of entanglement entropy signatures quantum phase transition in the $q$-deformed model.

\section{SUMMARY}

In summary we have studied the entanglement entropy in a class of quasi-symmetric multi-qubit quantum states. The entanglement entropy is obtained mainly by mapping the $q$-spin angular momentum decomposition of $q$-Dicke states to the Schmidt decomposition of multi-qubit states and is largely in parallel with the undeformed case. The main effect of $q$-deformation on entanglement is that it extrudes the entanglement to certain regions of the system. An implication of the result is that one could use the deformation parameter to modulate the distribution of entanglement in certain systems. In future works, one may 
also study the corresponding LOCC families [8], geometric entanglement and the Majorana representation [9] of the quasi-symmetric states, which would all together help to gain better understanding of entanglement structures in symmetric states. In addition we have studied entanglement entropy in a $q$-deformed LMG model related to these states. This model is a rare example in contrast with the bulk of models have been studied so far, which is not translation invariant and highly nonlinear. In this regard, it is remarkable that the singularities of the entanglement entropy is still able to detect the quantum phase transition point.

\section{Acknowledgements}

This work is supported by National Natural Science Foundation of China under Grant No. 11375168.

\section{References}

[1] M. A. Nielsen and I. L. Chuang, Quantum computation and quantum information (Cambridge university press, 2010).

[2] W. Dür, G. Vidal, and J. I. Cirac, Phys. Rev. A 62, 062314 (2000).

[3] X. Wang and K. Mølmer, Eur. Phys. J. D 18, 385 (2002).

[4] J. K. Stockton, J. Geremia, A. C. Doherty, and H. Mabuchi, Phys. Rev. A 67, 022112 (2003).

[5] X. Wang and B. C. Sanders, Phys. Rev. A 68, 012101 (2003).

[6] T.-C. Wei and P. M. Goldbart, Phys. Rev. A 68, 042307 (2003).

[7] A. U. Devi, R. Prabhu, and A. Rajagopal, Phys. Rev. Lett. 98, 060501 (2007).

[8] T. Bastin, S. Krins, P. Mathonet, M. Godefroid, L. Lamata, and E. Solano, Phys. Rev. Lett. 103, 070503 (2009).

[9] M. Aulbach, D. Markham, and M. Murao, New J. Phys. 12, 073025 (2010).

[10] H. J. Lipkin, N. Meshkov, and A. Glick, Nucl. Phys. 62, 188 (1965).

[11] T. J. Osborne and M. A. Nielsen, Phys. Rev. A 66, 032110 (2002).

[12] A. Osterloh, L. Amico, G. Falci, and R. Fazio, Nature 416, 608 (2002). 
[13] G. Vidal, J. I. Latorre, E. Rico, and A. Kitaev, Phys. Rev. Lett. 90, 227902 (2003).

[14] B.-Q. Jin and V. Korepin, J. Stat. Mech. Theor. Exp. 116, 79 (2004).

[15] P. Calabrese and J. Cardy, J. Stat. Mech. Theor. Exp. 2004, P06002 (2004).

[16] L. Amico, R. Fazio, A. Osterloh, and V. Vedral, Rev. Mod. Phys. 80, 517 (2008).

[17] J. I. Latorre, R. Orús, E. Rico, and J. Vidal, Phys. Rev. A 71, 64101 (2005).

[18] J. Vidal, G. Palacios, and R. Mosseri, Phys. Rev. A 69, 022107 (2004).

[19] J. Vidal, R. Mosseri, and J. Dukelsky, Phys. Rev. A 69, 054101 (2003).

[20] J. Vidal, S. Dusuel, and T. Barthel, J. Stat. Mech. Theor. Exp. 2007, P01015 (2007).

[21] H. Wichterich, J. Vidal, and S. Bose, Phys. Rev. A 81, 032311 (2010).

[22] X.-x. Zhang and F.-l. Li, Phys. Lett. A 377, 1053 (2013), ISSN 0375-9601.

[23] R. Prevedel, G. Cronenberg, M. S. Tame, M. Paternostro, P. Walther, M.-S. Kim, and A. Zeilinger, Phys. Rev. Lett. 103, 020503 (2009).

[24] W. Wieczorek, R. Krischek, N. Kiesel, P. Michelberger, G. Tóth, and H. Weinfurter, Phys. Rev. Lett. 103, 020504 (2009).

[25] V. Drinfeld, Quantum Groups pp. 798-819 (1987).

[26] M. Jimbo, Lett. Math. Phys. 10, 63 (1985).

[27] C. Kassel, Ollman, and Park, Quantum groups, vol. 155 (Springer New York, 1995).

[28] K. Berrada, A. Benmoussa, and Y. Hassouni, Quant. Inf. Proc. 10, 575 (2011).

[29] K. Berrada, M. El Baz, H. Eleuch, and Y. Hassouni, Quant. Inf. Proc. 11, 351 (2012).

[30] R. A. Santos, F. N. Paraan, V. E. Korepin, and A. Klümper, EPL 98, 37005 (2012).

[31] R. A. Santos, F. N. Paraan, V. E. Korepin, and A. Klümper, J. Phys. A: Math. Theor. 45, $175303(2012)$.

[32] B. Liu, K. Xue, G. Wang, C. Sun, L. Gou, and G. Du, Int. J. Quant. Inf. 11, 1350018 (2013).

[33] One may alternatively define (4) such that the power on $q$ is higher if more 1's are distributed in the leftmost positions or simply change $q \rightarrow q^{-1}$.

[34] R. Unanyan, C. Ionescu, and M. Fleischhauer, arXiv preprint quant-ph/0412164 (2004).

[35] A. Kirillov, J. Sov. Math. 53, 264 (1991).

[36] S. Avancini, A. Eiras, D. Galetti, B. Pimentel, and C. Lima, J. Phys. A: Math. Gen. 28, 4915 (1995). 